Review Article

\title{
Based on Network Pharmacology to Explore the Potential Bioactive Compounds and Mechanisms of Zuojin Pill for the Treatment of Ulcerative Colitis
}

\author{
Ying Wei $\mathbb{D}^{1,2}$ Sichen Ren $\mathbb{D}^{1,},{ }^{1,2}$ Ruilin Wang, ${ }^{3}$ Manyi Jing, ${ }^{2}$ Honghong Liu $\mathbb{D},{ }^{3}$ Min Wang, \\ Hongtao Song $\mathbb{D D}^{4}{ }^{4}$ and Yanling Zhao ${ }^{2}{ }^{2}$ \\ ${ }^{1}$ School of Pharmacy, Chengdu University of Traditional Chinese Medicine, Chengdu, China \\ ${ }^{2}$ Department of Pharmacy, The Fifth Medical Center of Chinese PLA General Hospital, Beijing, China \\ ${ }^{3}$ China Military Institute of Chinese Medicine, The Fifth Medical Center of Chinese PLA General Hospital, Beijing, China \\ ${ }^{4}$ Department of Pharmacy, 900th Hospital of the Joint Logistics Team, Fuzhou, China
}

Correspondence should be addressed to Hongtao Song; sohoto@vip.163.com and Yanling Zhao; zhaoyl2855@126.com

Received 12 May 2021; Revised 6 August 2021; Accepted 16 August 2021; Published 27 August 2021

Academic Editor: Ivan Luzardo-Ocampo

Copyright (c) 2021 Ying Wei et al. This is an open access article distributed under the Creative Commons Attribution License, which permits unrestricted use, distribution, and reproduction in any medium, provided the original work is properly cited.

Background. Zuojin Pill (ZJP), a classic prescription, has the potential to prevent ulcerative colitis (UC). However, the active components and mechanisms of ZJP are still arcane. This study aimed to use a network pharmacology approach to find the bioactive compounds and potential action mechanisms of ZJP in the treatment of UC. Methods. Firstly, the components and putative targets of ZJP were collected based on herbal medicine target databases, and a network containing the interaction between the targets of ZJP and the potential therapeutic targets of UC was established. Then, topological parameters were calculated to identify the key targets in the network and, in turn, to import them into the David database to perform path enrichment analysis. Results. 14 potential therapeutic components of ZJP and 26 key targets were obtained. These targets were related to signal transduction, MAPK cascade, inflammatory response, immune response, and the apoptotic process of UC. Moreover, the PI3KAkt signaling pathway, MAPK signaling pathway, toll-like receptor signaling pathway, and Prolactin signaling pathway were predicted to participate in ZJP treating UC. Among them, 14 active components of ZJP directly regulate these pathways. Conclusion. ZJP could alleviate UC through the predicted components and mechanisms. The 14 predicted active components of ZJP may mainly play a therapeutic role for UC through synergistic regulation of the PI3K-Akt signaling pathway and MAPK signaling pathway.

\section{Introduction}

Ulcerative colitis (UC) is a chronic nonspecific inflammatory disease. It was listed as a modern refractory disease by the World Health Organization because of its unclear etiology, high clinical recurrence rate, and association with colon cancer [1]. In recent years, the incidence rate of UC has been increasing worldwide [2]. Aminosalicylic acids, hormones, and immunosuppressants are mainly used in the clinical treatment of UC, but there are obvious adverse reactions. For example, mesalazine is the first-line drug in the treatment of UC, but its long-term application can damage the liver and kidney function of patients [3]. Therefore, many patients with UC as well as physicians and researchers are increasingly considering complementary and alternative medicine options $[4,5]$.

Zuojin Pill (ZJP), a classic prescription for gastrointestinal diseases, has been widely used in the clinical treatment of gastrointestinal diseases since ancient times in China because of its safety and effectiveness. It composes of Coptis chinensis Franch. (Ranunculaceae, recorded in the Chinese Pharmacopoeia as Rhizoma Coptidis) and Evodia rutaecarpa (Juss.) Benth. (Rutaceae, recorded in the Chinese Pharmacopoeia as Fructus Evodiae) $(6: 1, \mathrm{~g} / \mathrm{g})$. 
In recent years, ZJP has been reported to have beneficial evidence on UC [6]. However, the potential active ingredients and molecular mechanism of ZJP on UC are hardly clarified. With the rapid development of bioinformatics, systems biology, and polypharmacology, the pharmacology method based on network pharmacology has been proved to be an effective means to explore the compatibility and mechanisms of traditional Chinese medicine [7-9].

Therefore, this study aimed to use a comprehensive network pharmacology-based approach to investigate the potential effective components and molecular mechanisms of ZJP in treating UC. The flowchart of the experimental procedures of our study is shown in Figure 1.

\section{Materials and Methods}

2.1. Chemical Components of ZJP. We searched the Traditional Chinese Medicine Systems Pharmacology Database and Analysis Platform (TCMSP, https://old.tcmsp-e.com/ tcmsp.php, updated on May 31, 2014), Chinese Academy of Sciences Chemistry database (CASC, http://www. organchem.csdb.cn/scdb/main/slogin.asp, updated on December 31, 2019), and related literatures to collect the chemical components of the two herbs contained in ZJP using "Coptidis Rhizoma" and "Fructus Evodiae" as the queries. The TCMSP is a unique systematic pharmacology database of Chinese herbal medicines, chemicals, targets, and drug-target networks. The CASC is one of the most comprehensive chemical databases in the world, which can provide chemical information of Chinese herbal medicine and natural products.

2.2. Candidate Targets of ZJP. The targets of chemical components in ZJP were mainly obtained from the TCMSP database. If the targets of chemical constituents reported in the literature were not included in the database, they were obtained through SwissTargetPrediction (http://www. swisstargetprediction.ch/).

2.3. Establishment of a Target Database for Treating UC. The known therapeutic targets of UC were acquired from the DisGeNET database (https://www.disgenet.org/) and Online Mendelian Inheritance in Man database (OMIM, https:// omim.org/). After screening the target (score $\geq 0.01$ ) and removing the duplicate value, they were collected and used for data analysis serving as a target database for treating UC.

2.4. Construction and Analysis of the PPI Network. The protein-protein interactions (PPIs) of each target were generated from the Search Tool for the Retrieval of Interacting Genes/Proteins (STRING) database (https://string$\mathrm{db}$.org/), and the interactions with a probabilistic association confidence score $\geq 0.9$ were selected in this study. All networks were constructed and analyzed by using Cytoscape v3.7.1. Three topological parameters were used as the screening criteria to obtain a hub network and key targets (degree centrality $\geq 2 \times$ median degree centrality; betweenness centrality $\geq$ median betweenness centrality; and closeness centrality $\geq$ median closeness centrality).

2.5. GO and KEGG Pathway Enrichment Analysis. Gene ontology (GO) analysis and Kyoto Encyclopedia of Genes and Genomes (KEGG) pathway enrichment analysis were performed using the Database for Annotation, Visualization, and Integrated Discovery (DAVID) (https://david.ncifcrf. gov/). GO terms and KEGG pathways with $p$ value $<0.05$ were considered statistically significant.

2.6. Construction and Analysis of the Component-TargetPathway Network. The component-target-pathway network was constructed and visualized via Cytoscape v3.7.1 to identify key and directly linked molecules and targets. The directly linked molecules were screened by oral bioavailability (OB) and drug-likeness (DL) (OB $\geq 30 \%, \mathrm{DL} \geq 0.18)$.

2.7. Toxicity Prediction of Components in ZJP. ADMETlab (http://admet.scbdd.com) is a free tool database for the prediction of absorption, distribution, metabolism, and excretion and various toxicities (ADMET) properties and has been widely used in chemical and pharmaceutical fields. Each SMILES of molecule was input to obtain organ toxicity and other toxic properties.

\section{Results}

3.1. Composite Ingredients of ZJP. A total of 161 chemical ingredients (Table S1) of the two herbal medicines in ZJP were collected from the TCMSP, CASC, and related literatures, including 32 ingredients in Rhizoma Coptidis and 129 ingredients in Fructus Evodiae. There were 5 shared components: berberine, obacunone, quercetin, isovanillin, and limonin (Figure 2(a)).

3.2. Putative Targets of Components. The targets of eight components (palmidin A, moupinamide, dehydroevodiamine, evodione, synephrine, obacunone, 13-methylmyristate, and cis-beta-ocimene) were predicted online by SwissTargetPrediction, and the targets of remaining compounds were from the TCMSP. Finally, 249 direct acting targets of Rhizoma Coptidis and 357 direct acting targets of Fructus Evodiae were obtained. After removing repeated targets, 386 targets were obtained for subsequent analysis. Also, there were 220 overlapping targets between the two herbs (Figure 2(b)), which suggested that there might be an interaction between Rhizoma Coptidis and Fructus Evodiae in the treatment process. The relationship between the 161 components and the 386 targets is shown in Figure 2(c).

3.3. The Known Therapeutic Targets of UC. 1458 targets of UC were obtained from the DisGeNET database, and 180 targets of UC were obtained from the OMIM database. A total of 1527 targets of UC were obtained after removing the repeats for subsequent analysis (Figure 3(a)). By comparing the components targets and the disease targets through the 


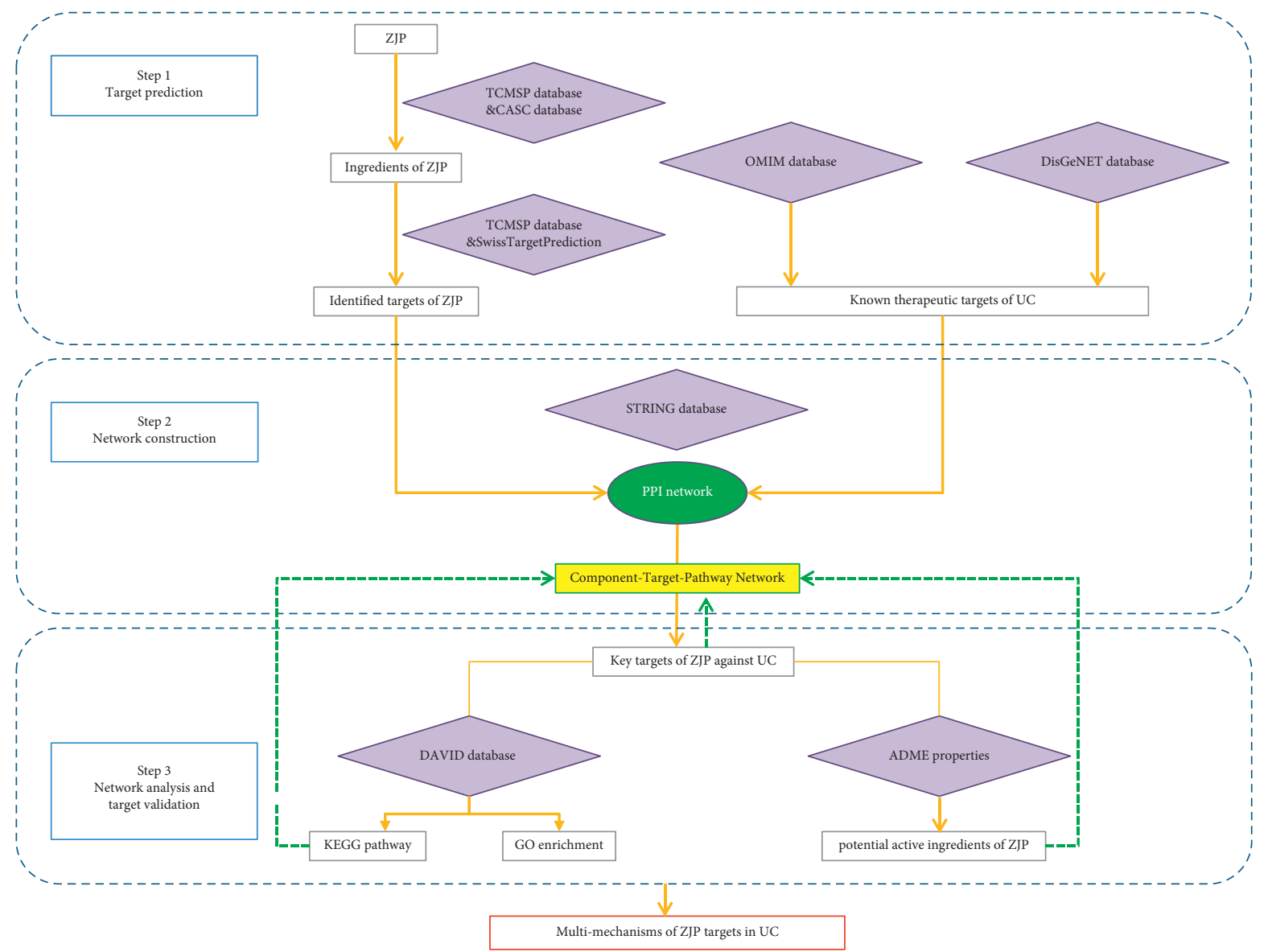

FIGURE 1: The flowchart of the network pharmacology-based strategy for deciphering the mechanisms of ZJP acting on UC. ZJP: Zuojin pill; UC: ulcerative colitis; TCMSP: Traditional Chinese Medicine Systems Pharmacology Database and Analysis Platform; CASC: Chinese Academy of Sciences Chemistry; OMIM: Online Mendelian Inheritance in Man; STRING: Search Tool for the Retrieval of Interacting Genes/Proteins; PPI: protein-protein interaction; DAVID: the Database for Annotation, Visualization, and Integrated Discovery; KEGG: Kyoto Encyclopedia of Genes and Genomes; GO: gene ontology; ADME: absorption, distribution, metabolism, and excretion.

Venn diagram, 146 targets for the treatment of UC were identified (Figure 3(b)). These targets may be the key proteins of ZJP in the treatment of UC.

3.4. The PPI Network of 146 Common Targets. The PPI network was constructed by STRING (confidence score$\geq 0.9$ ), with 125 nodes and 645 edges (Figure 4). Based on the topological parameters, we identified 26 key targets, including JUN, MAPK1, TNF, PIK3CA, RELA, AKT1, TP53, FOS, SRC, IL6, MAPK14, MAPK8, VEGFA, CTNNB1, EGFR, SP1, ESR1, CXCL8, MYC, IL1B, JAK2, IL2, IL4, SMAD3, STAT1, and NR3C1 (Table 1).

3.5. GO and KEGG Enrichment Analysis. To investigate the biological functions and pathway of the key targets of ZJP, the gene ontology (GO), biological process (BP), and KEGG were performed through the functional annotation tool of DAVID and Cytoscape3.7.1. The top $10 \mathrm{GO}$ terms and pathways were significantly enriched, respectively (Figures 5 and 6 ).

In UC, the BPs mainly regulated by ZJP were signal transduction, response to drug, cellular response to lipopolysaccharide, MAPK cascade, inflammatory response, immune response, transcription from RNA polymerase II promoter, apoptotic process, regulation of sequence-specific DNA binding transcription factor activity, and lipopolysaccharide-mediated signaling pathway (Table 2).

The pathways mainly regulated by ZJP were pathways in cancer, Chagas disease, hepatitis B, the Toll-like receptor signaling pathway, influenza $A$, proteoglycans in cancer, the MAPK signaling pathway, HTLV-I infection, the PI3K-Akt signaling pathway, and the prolactin signaling pathway (Table 3). Many of these enrichment pathways are related to other pathological effects, which may be due to the similar molecular targets of different diseases. In other words, the same molecular targets may be involved in different pathological processes of diseases. Among them, the changes of the Toll-like receptor signaling pathway, MAPK signaling pathway, and PI3K-Akt signaling pathway are most closely related to UC.

3.6. Component-Target-Pathway Network. The key targets of ZJP enriched to four signaling pathways are shown in $\mathrm{Ta}-$ ble 3. We constructed the "component-target-pathway" 


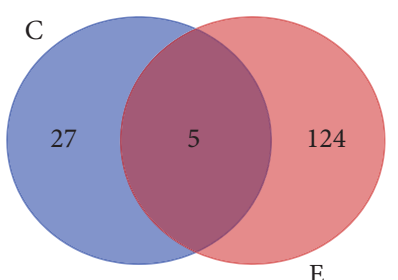

(a)

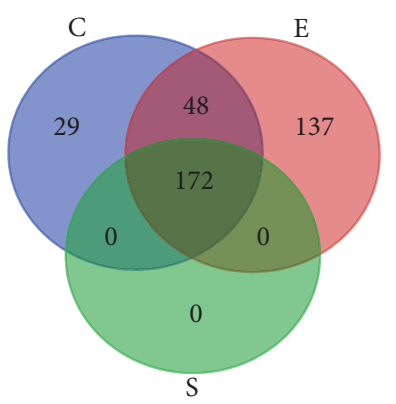

(b)

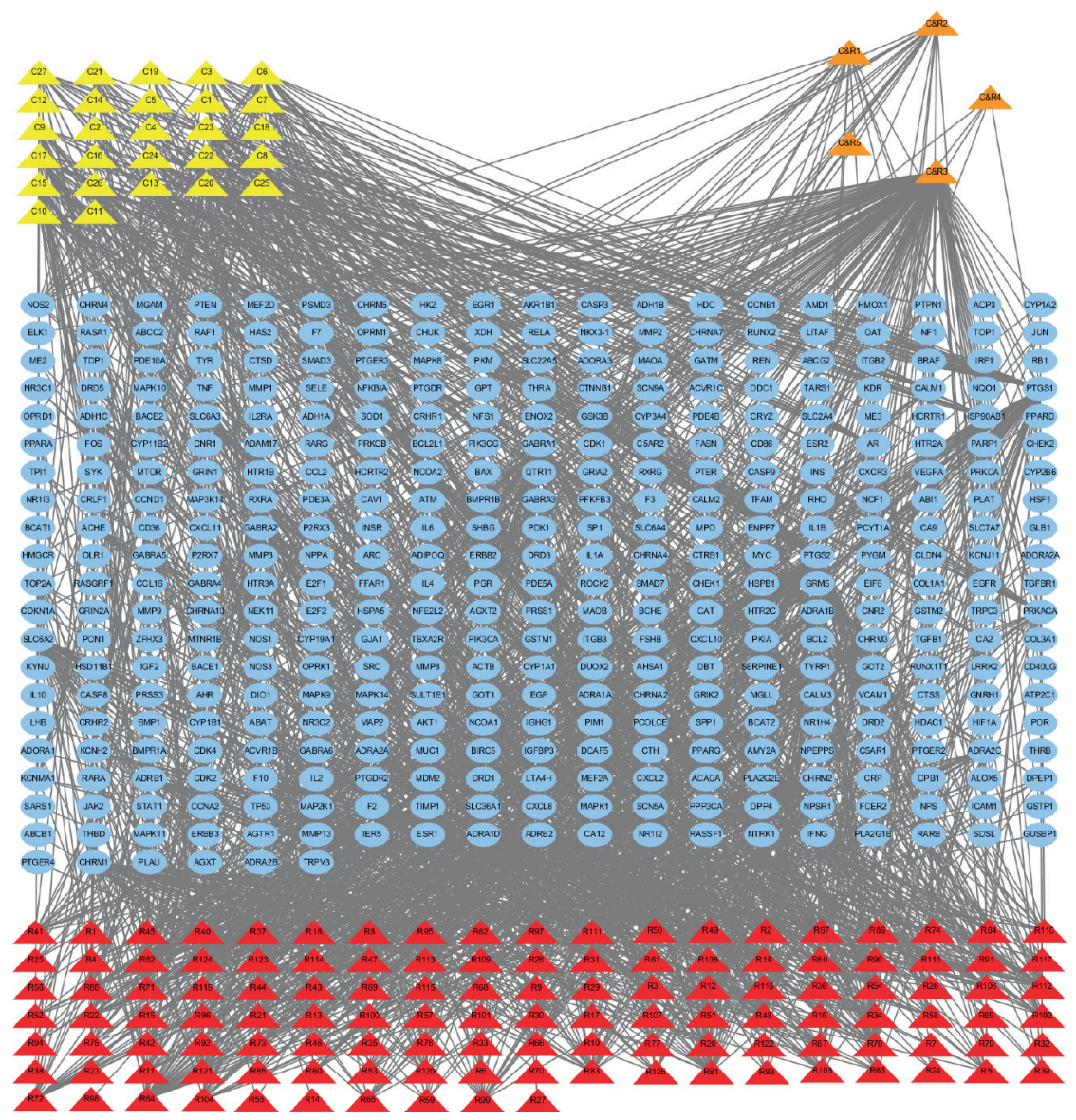

(c)

Figure 2: Ingredients and targets of ZJP. (a) Distribution of active compounds among the herbs (C: Rhizoma Coptidis ingredients; E: Fructus Evodiae ingredients). (b) Distribution of potential targets among the herbs (C: Rhizoma Coptidis targets; E: Fructus Evodiae targets; and S: Shared targets). (c) Herb-compound-target network of ZJP (the triangles represent components of ZJP, 32 yellow triangles represent Rhizoma Coptidis components, 127 red triangles represent Rhizoma Coptidis components, 5 orange triangles represent shared components, and blue circles represent the 386 potential targets of ZJP).

network with 14 directly linked components screened by ADME, 19 directly regulated targets, and four UC-related signaling pathways (Figure 7). The network showed that these 14 molecules were involved in regulating the toll-like receptor signaling pathway, MAPK signaling pathway, prolactin signaling pathway, and PI3K-Akt signaling pathway. In this network, we identified directed molecules and targets (Table 4). In order to further characterize the safety of these active ingredients, toxicity prediction of 14 active components in ZJP is shown in Table 5. 


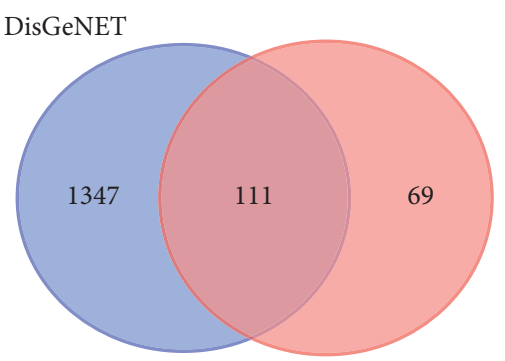

OMIM

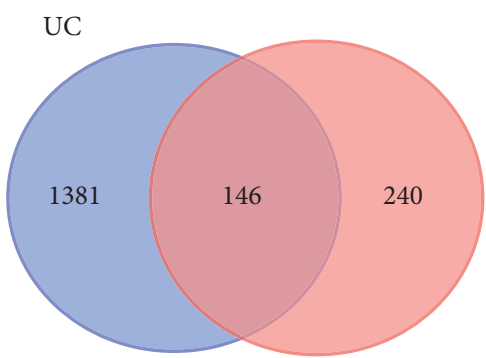

ZJP

(a)

(b)

FIgURE 3: Distribution of the therapeutic targets of UC. (a) Distribution of the known therapeutic targets of UC from two databases. (b) Distribution of ZJP targets and the disease targets. ZJP: Zuojin pill; UC: ulcerative colitis; and OMIM: Online Mendelian Inheritance in Man.

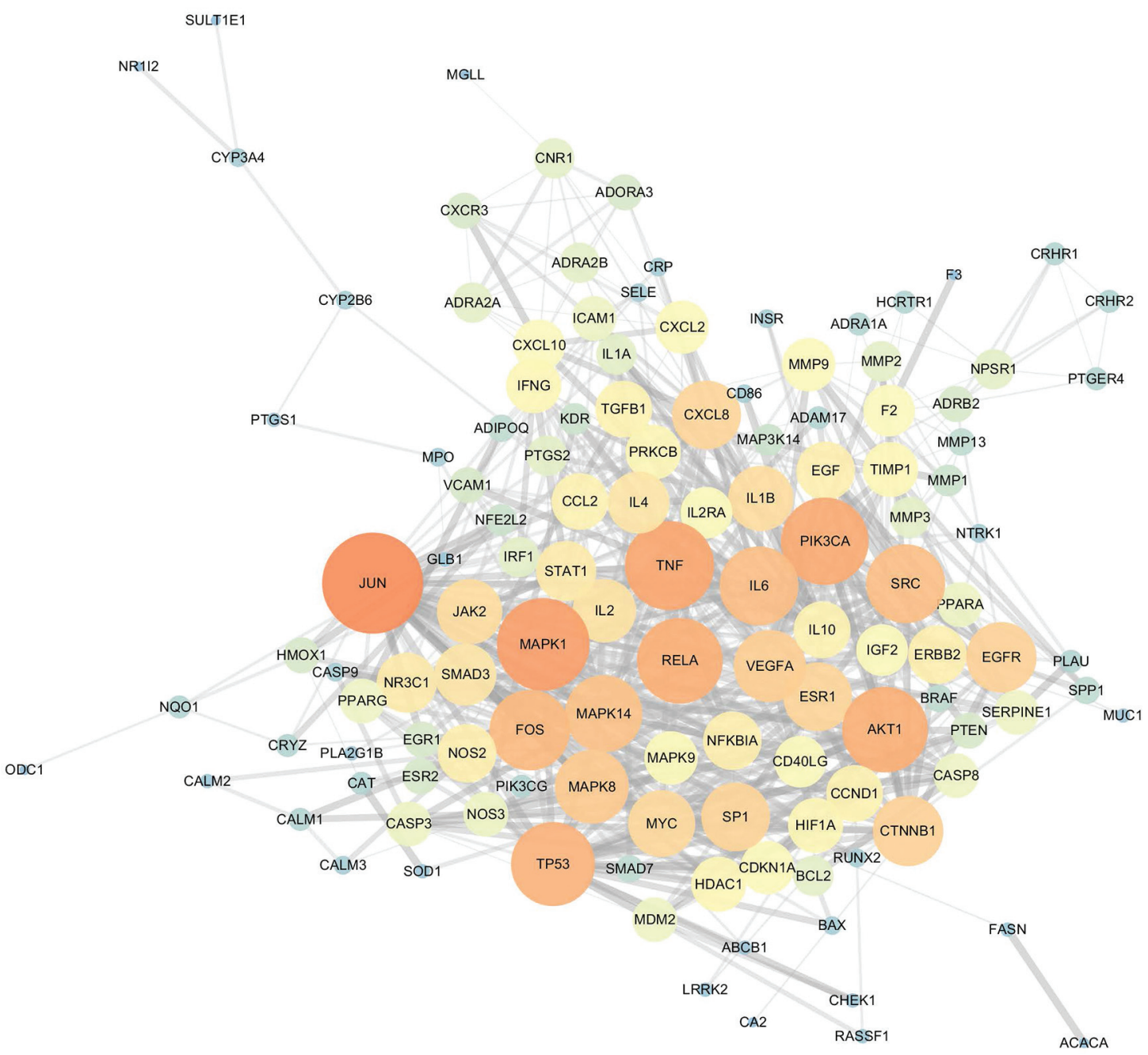

FIgURE 4: The PPI network of 146 common targets. The higher the degree value is, the brighter the color is and the larger the node is.

\section{Discussion}

The pathogenesis of UC is sophisticated, including genetic, environmental, psychological stress, and other factors [1]. In view of the complicated pathogenesis, a single target drug hardly obtains better therapeutic effect, while traditional Chinese medicine (TCM) with multitarget effect has become a considerable source of UC therapeutic drugs $[10,11]$. 
TABle 1: Hub genes in the PPI network.

\begin{tabular}{|c|c|c|c|}
\hline Gene name & Degree & Betweenness centrality & Closeness centrality \\
\hline JUN & 40 & 0.0866053 & 0.52765957 \\
\hline MAPK1 & 35 & 0.10522212 & 0.51239669 \\
\hline TNF & 33 & 0.06737271 & 0.49206349 \\
\hline PIK3CA & 32 & 0.09809998 & 0.49011858 \\
\hline RELA & 31 & 0.04010596 & 0.50612245 \\
\hline AKT1 & 31 & 0.07469638 & 0.49011858 \\
\hline TP53 & 30 & 0.06701158 & 0.49011858 \\
\hline FOS & 28 & 0.03619664 & 0.484375 \\
\hline SRC & 27 & 0.05381998 & 0.496 \\
\hline IL6 & 27 & 0.05383839 & 0.484375 \\
\hline MAPK14 & 26 & 0.02930984 & 0.47328244 \\
\hline MAPK8 & 24 & 0.0236753 & 0.46096654 \\
\hline VEGFA & 23 & 0.05077093 & 0.5 \\
\hline CTNNB1 & 22 & 0.03781139 & 0.46268657 \\
\hline EGFR & 22 & 0.05992162 & 0.48627451 \\
\hline SP1 & 21 & 0.03912481 & 0.46441948 \\
\hline ESR1 & 21 & 0.00980253 & 0.47148289 \\
\hline CXCL8 & 21 & 0.06791767 & 0.44604317 \\
\hline MYC & 20 & 0.00939055 & 0.46969697 \\
\hline IL1B & 19 & 0.01687947 & 0.46441948 \\
\hline JAK2 & 19 & 0.01407263 & 0.45925926 \\
\hline IL2 & 18 & 0.00668683 & 0.455888235 \\
\hline IL4 & 17 & 0.01066433 & 0.44765343 \\
\hline SMAD3 & 17 & 0.01141516 & 0.43055556 \\
\hline STAT1 & 16 & 0.01339176 & 0.44927536 \\
\hline NR3C1 & 16 & 0.00783583 & 0.44765343 \\
\hline
\end{tabular}

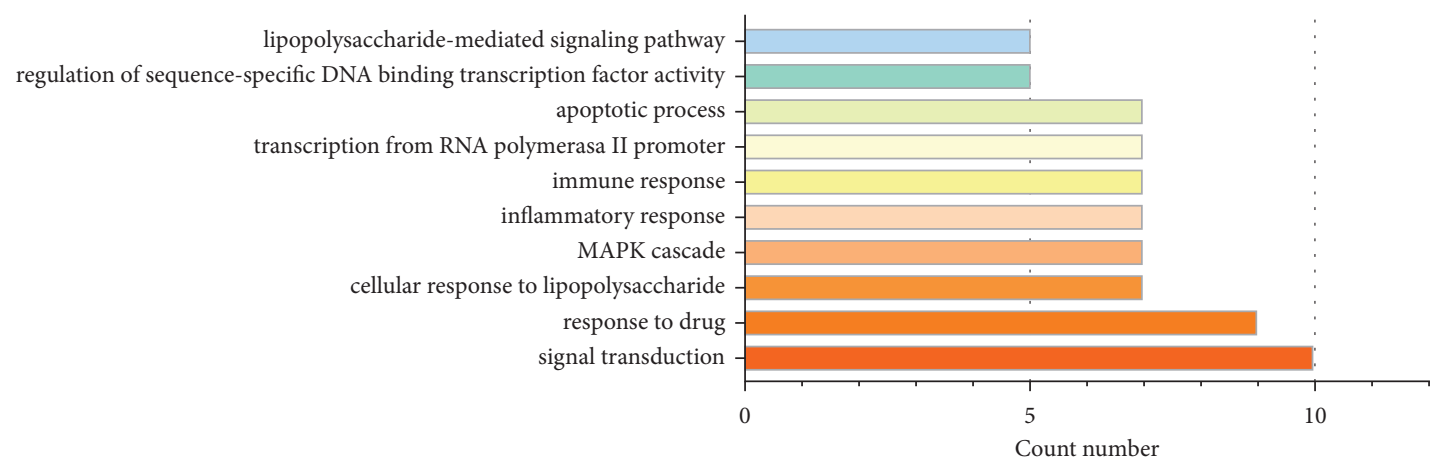

Figure 5: Top $10 \mathrm{GO}$ terms of hub genes.

Moreover, network pharmacology provides a new strategy for the research of TCM through exploring the relationship between drugs and diseases from a holistic perspective.

In this study, we successfully identified 14 active molecules and 3 important pathways related to UC through network pharmacology and bioinformatics. This method can more clearly identify the targets and specific mechanisms of TCM in the treatment of diseases and obtain the key active molecules, which is of great significance for the development of natural drugs and the treatment of diseases.

GO-BP enrichment analysis showed that ZJP may play an anti-UC role by regulating inflammatory response, immune response, apoptosis, MAPK cascade, signal transduction, and other biological functions. Pathway enrichment analysis results showed that many other diseases were enriched in addition to UC, which may be because the same molecular targets exist in different pathological processes of diseases. This is not only the limitation of network pharmacology but also the divergence points of the research direction. Therefore, we selected the enrichment pathway closely related to UC in our research. Finally, based on the two aspects of network construction and central network evaluation, 26 key targets of the ZJP potential target network were selected which were significantly enriched in three UCrelated signaling pathways, including the PI3K-Akt signaling pathway, MAPK signaling pathway, and toll-like receptor signaling pathway. Studies have shown that the PI3K-Akt signaling pathway could regulate the inflammation and oxidative stress of UC $[12,13]$; the MAPK pathway played a key role in regulating the development of UC inflammation 


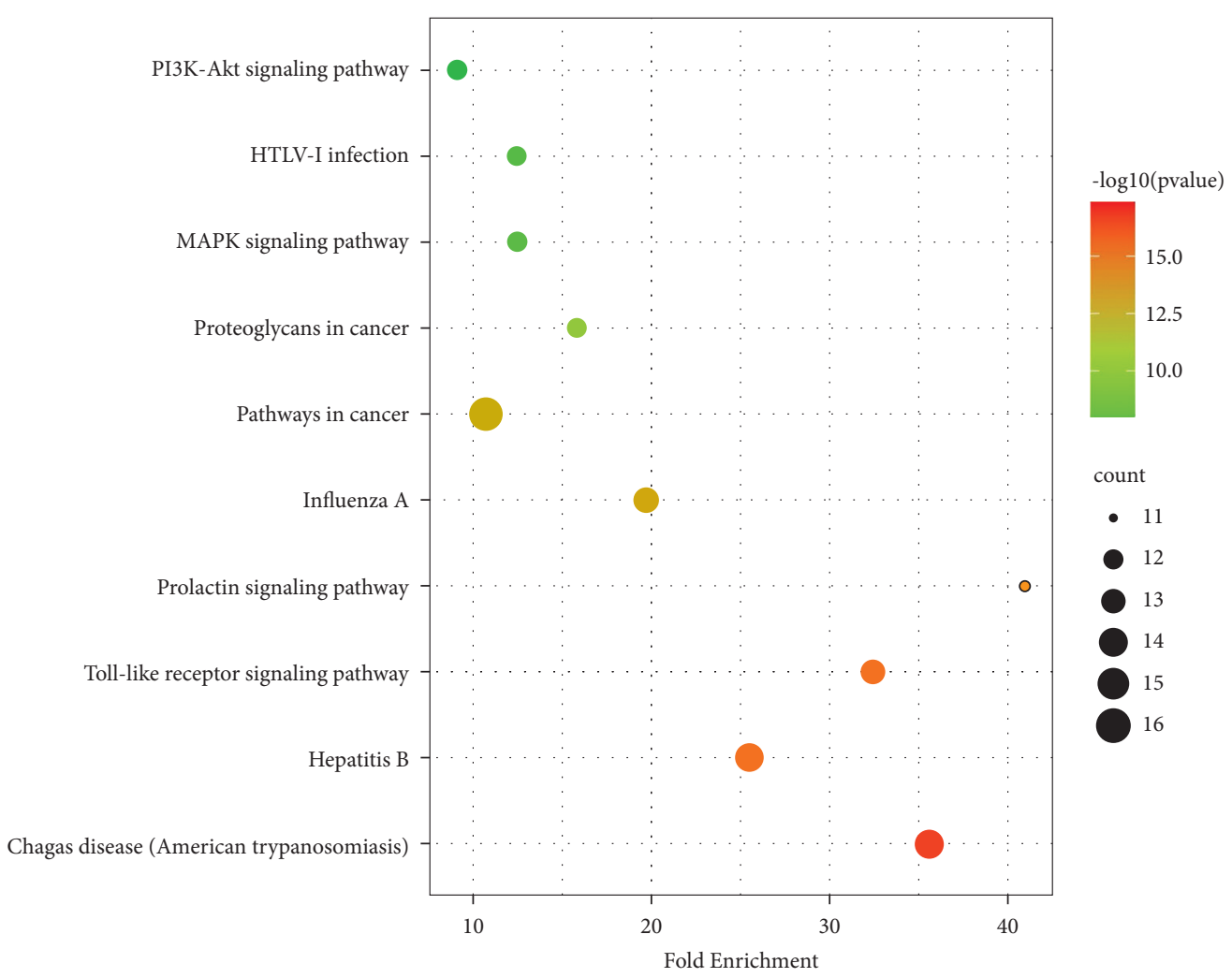

Figure 6: Top 10 KEGG terms of hub genes.

TABle 2: Top 10 GO terms of hub genes.

\begin{tabular}{|c|c|c|c|}
\hline Term & Count & $\%$ & $p$ value \\
\hline Signal transduction & 10 & 38.46 & $2.60 \mathrm{E}-05$ \\
\hline Response to drug & 9 & 34.62 & 8.71E-09 \\
\hline Cellular response to lipopolysaccharide & 7 & 26.92 & $1.30 \mathrm{E}-08$ \\
\hline MAPK cascade & 7 & 26.92 & $1.88 \mathrm{E}-06$ \\
\hline Inflammatory response & 7 & 26.92 & $1.56 \mathrm{E}-05$ \\
\hline Immune response & 7 & 26.92 & $2.83 \mathrm{E}-05$ \\
\hline Transcription from RNA polymerase II promoter & 7 & 26.92 & $8.52 \mathrm{E}-05$ \\
\hline Apoptotic process & 7 & 26.92 & $1.48 \mathrm{E}-04$ \\
\hline Regulation of sequence-specific DNA binding transcription factor activity & 5 & 19.23 & 4.73E-08 \\
\hline Lipopolysaccharide-mediated signaling pathway & 5 & 19.23 & $1.34 \mathrm{E}-07$ \\
\hline
\end{tabular}

[14]; the toll-like receptor signaling pathway played an important role in the immune system and participated in the inflammatory process of UC [15]. However, the key target of this study enriched in the toll-like receptor signaling pathway was focused on the downstream MAPK pathway crosstalk. Thereby, it could be considered that ZJP treatment of UC mainly directly regulated the PI3K-Akt signaling pathway and MAPK signaling pathway. Figures 8-10 summarize the representative pathways in the progress of UC and the key targets (red nodes) related to ZJP, indicating that ZJP treatment of UC may be closely related to the regulation of inflammation and cell proliferation.

Among the 14 predicted active components, 9 compounds such as berberine, obacunone, quercetin, coptisine derivatives, berberrubine, palmatine, beta sitosterol, isorhamnetin, and rutaecarpine have been proved to be effective in the treatment of UC, and the related mechanism of some compounds was consistent with the predicted pathway in this study. Studies have shown that berberine could inhibit the p38 MAPK signaling pathway and decrease the levels of inflammatory biomarkers IL-1, TNF- $\alpha$, and IL-6 in UC [16]; obacunone could protect against UC in mice by modulating gut microbiota, attenuating TLR4/NF- $\kappa \mathrm{B}$ signaling cascades, and improving disrupted epithelial barriers [17]; quercetin could inhibit the release of proinflammatory mediators and the expression of inflammatory proteins in UC [18]; coptisine derivatives such as dihydrocoptisine, quaternary coptisines, and tetrahydrocoptisines were proved to have anti-UC activities, among which dihydroberberine has better development value [19]; berberrubine could attenuate mucosal lesions and inflammation in UC [20]; palmatine could attenuate dextran sulfate sodium- (DSS-) 
TAвLE 3: Top 10 KEGG pathways of hub genes.

\begin{tabular}{|c|c|c|c|c|}
\hline Term & it & $\%$ & value & Genes \\
\hline Pathways in cancer & 16 & 61.54 & $\begin{array}{c}3.36 \mathrm{E}- \\
13\end{array}$ & $\begin{array}{l}\text { JUN, SMAD3, CXCL8, STAT1, FOS, EGFR, RELA, VEGFA, IL6, MAPK8, PIK3CA, } \\
\text { MYC, AKT1, MAPK1, CTNNB1, and TP53 }\end{array}$ \\
\hline Chagas disease & 14 & 53.85 & $\begin{array}{l}4.42 \mathrm{E}- \\
18\end{array}$ & $\begin{array}{l}\text { JUN, SMAD3, CXCL8, FOS, MAPK14, TNF, IL2, RELA, IL6, MAPK8, PIK3CA, } \\
\text { IL1B, AKT1, and MAPK1 }\end{array}$ \\
\hline Hepatitis B & 14 & 53.85 & $\begin{array}{c}3.88 \mathrm{E}- \\
16\end{array}$ & $\begin{array}{c}\text { JUN, CXCL8, STAT1, SRC, FOS, TNF, RELA, IL6, MAPK8, PIK3CA, MYC, AKT1, } \\
\text { MAPK1, and TP53 }\end{array}$ \\
\hline $\begin{array}{l}\text { Toll-like } r \\
\text { pathway }\end{array}$ & 13 & 50.00 & $\begin{array}{l}4.12 \mathrm{E}- \\
16\end{array}$ & $\begin{array}{l}\text { JUN, CXCL8, STAT1, FOS, MAPK14, TNF, RELA, IL6, MAPK8, PIK3CA, IL1B, } \\
\text { AKT1, and MAPK1 }\end{array}$ \\
\hline Influ & 13 & 50.00 & $\begin{array}{l}1.84 \mathrm{E}- \\
13\end{array}$ & $\begin{array}{c}\text { JUN, CXCL8, STAT1, MAPK14, TNF, RELA, IL6, MAPK8, PIK3CA, IL1B, AKT1, } \\
\text { MAPK1, and JAK2 }\end{array}$ \\
\hline Prote & 12 & 46.15 & $\begin{array}{c}2.98 \mathrm{E}- \\
11\end{array}$ & $\begin{array}{c}\text { PIK3CA, SRC, MYC, MAPK1, CTNNB1, AKT1, MAPK14, ESR1, TNF, TP53, } \\
\text { EGFR, and VEGFA }\end{array}$ \\
\hline MAP & 12 & 46.15 & $\begin{array}{c}3.79 \mathrm{E}- \\
10\end{array}$ & $\begin{array}{c}\text { JUN, MAPK8, MYC, IL1B, MAPK1, AKT1, FOS, MAPK14, TNF, TP53, RELA, } \\
\text { EGFR }\end{array}$ \\
\hline HTL & 12 & 46.15 & $\begin{array}{c}3.96 \mathrm{E}- \\
10\end{array}$ & $\begin{array}{l}\text { IL6, JUN, SMAD3, PIK3CA, MYC, CTNNB1, AKT1, FOS, TNF, TP53, RELA, and } \\
\text { IL2 }\end{array}$ \\
\hline $\begin{array}{l}\text { PI3K } \\
\text { path }\end{array}$ & 12 & 46.15 & $\begin{array}{c}1.02 \mathrm{E}- \\
08\end{array}$ & $\begin{array}{l}\text { IL4, IL6, PIK3CA, MYC, MAPK1, AKT1, JAK2, TP53, RELA, EGFR, IL2, and } \\
\text { VEGFA }\end{array}$ \\
\hline Prolactin signaling pathway & 11 & 42.31 & $\begin{array}{c}2.05 \mathrm{E}- \\
14\end{array}$ & $\begin{array}{c}\text { MAPK8, PIK3CA, STAT1, SRC, MAPK1, AKT1, FOS, JAK2, MAPK14, ESR1, and } \\
\text { RELA }\end{array}$ \\
\hline
\end{tabular}

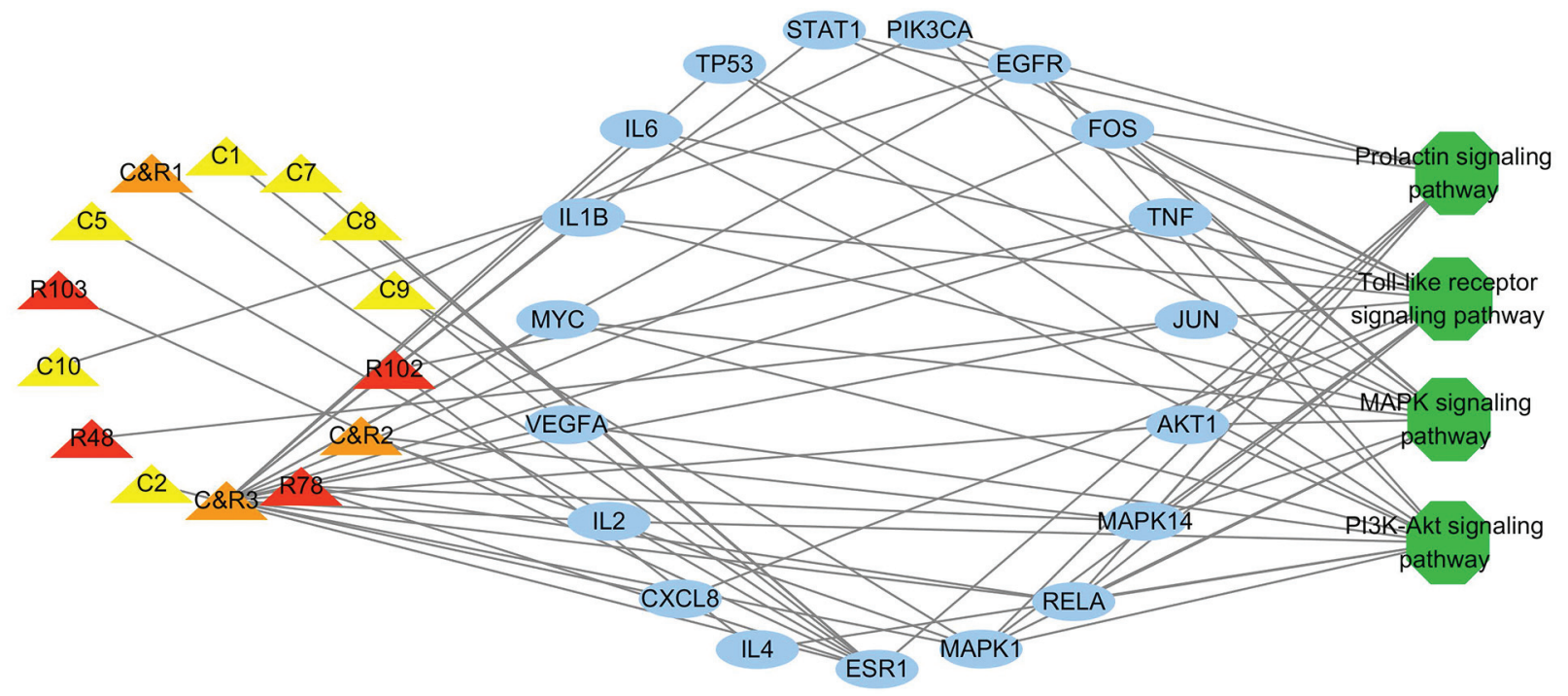

FIGURE 7: Component-target-pathway network. The triangles represent components of ZJP, 7 yellow triangles represent Rhizoma Coptidis (C) components, red triangles represent Fructus Evodiae (R) components, 3 orange triangles represent shared (C\&R) components, 19 blue circles represent the potential targets of ZJP, and 4 green octagons represent UC-related signaling pathways.

TABLE 4: Directly linked components screened by OB and DL.

\begin{tabular}{|c|c|c|c|c|}
\hline Code name & Molecule name & MW & OB (\%) & $\overline{\mathrm{DL}}$ \\
\hline C\&R1 & Berberine & 336.39 & 36.86 & 0.78 \\
\hline C\&R2 & Obacunone & 454.56 & 43.29 & 0.77 \\
\hline C\&R3 & Quercetin & 302.25 & 46.43 & 0.28 \\
\hline $\mathrm{C} 1$ & Worenine & 334.37 & 45.83 & 0.87 \\
\hline $\mathrm{C} 2$ & Coptisine & 320.34 & 30.67 & 0.86 \\
\hline $\mathrm{C} 5$ & Epiberberine & 336.39 & 43.09 & 0.78 \\
\hline $\mathrm{C} 7$ & Berberrubine & 322.36 & 35.74 & 0.73 \\
\hline $\mathrm{C} 8$ & Palmatine & 510.52 & 35.36 & 0.65 \\
\hline $\mathrm{C} 9$ & Palmidin A & 510.52 & 35.36 & 0.65 \\
\hline $\mathrm{C} 10$ & Moupinamide & 313.38 & 86.71 & 0.26 \\
\hline
\end{tabular}


TABLE 4: Continued.

\begin{tabular}{|c|c|c|c|c|}
\hline Code name & Molecule name & MW & OB (\%) & $\mathrm{DL}$ \\
\hline R48 & Beta-sitosterol & 414.79 & 36.91 & 0.75 \\
\hline R78 & Isorhamnetin & 316.28 & 49.6 & 0.31 \\
\hline R102 & Rutaecarpine & 287.34 & 40.3 & 0.6 \\
\hline R103 & Rutalinidine & 275.33 & 40.89 & 0.22 \\
\hline
\end{tabular}

Abbreviations: C: Rhizoma Coptidis components; R: Fructus Evodiae components; C\&R: Rhizoma Coptidis and Fructus Evodiae shared components.

TABLE 5: Toxicity prediction of 14 active components in ZJP.

\begin{tabular}{lccccc}
\hline Code name & Molecule name & Ames mutagenesis & Hepatotoxicity & Acute oral toxicity & Acute oral toxicity evaluation \\
\hline CR1 & Berberine & --- & ++ & $551.84 \mathrm{mg} / \mathrm{kg}$ & Low \\
CR2 & Obacunone & -- & + & $51.827 \mathrm{mg} / \mathrm{kg}$ & Toxicity \\
CR3 & Quercetin & ++ & + & $698.794 \mathrm{mg} / \mathrm{kg}$ & Low \\
C1 & Worenine & - & + & $558.731 \mathrm{mg} / \mathrm{kg}$ & Low \\
C2 & Coptisine & - & - & $547.759 \mathrm{mg} / \mathrm{kg}$ & Low \\
C5 & Epiberberine & --- & ++ & $571.233 \mathrm{mg} / \mathrm{kg}$ & Low \\
C7 & Berberrubine & --- & ++ & $301.519 \mathrm{mg} / \mathrm{kg}$ & Toxicity \\
C8 & Palmatine & - & ++ & $660.767 \mathrm{mg} / \mathrm{kg}$ & Low \\
C9 & Palmidin A & + & --- & $147.569 \mathrm{mg} / \mathrm{kg}$ & Toxicity \\
C10 & Moupinamide & - & ++ & $1603.37 \mathrm{mg} / \mathrm{kg}$ & Low \\
R48 & Beta-sitosterol & --- & --- & $273.371 \mathrm{mg} / \mathrm{kg}$ & Toxicity \\
R78 & Isorhamnetin & --- & + & $604.02 \mathrm{mg} / \mathrm{kg}$ & Low \\
R102 & Rutaecarpine & --- & ++ & $624.265 \mathrm{mg} / \mathrm{kg}$ & Low \\
R103 & Rutalinidine & - & +++ & $591.306 \mathrm{mg} / \mathrm{kg}$ & Low \\
\hline
\end{tabular}

Note. The "+" and "-" represent the predicted toxicity possibility. $0.1(---)$; 01-0.3(--); 0.3-05(-); 0.5-0.7(+); 0.7-0.9(++); 0.9-10(+++). Acute oral toxicity evaluation involves high toxicity $(1 \sim 50 \mathrm{mg} / \mathrm{kg})$, toxicity $(51 \sim 500 \mathrm{mg} / \mathrm{kg})$, and low toxicity $(501 \sim 5000 \mathrm{mg} / \mathrm{kg})$. C: Rhizoma Coptidis components; R: Fructus Evodiae components; C\&R: Rhizoma Coptidis and Fructus Evodiae shared components.

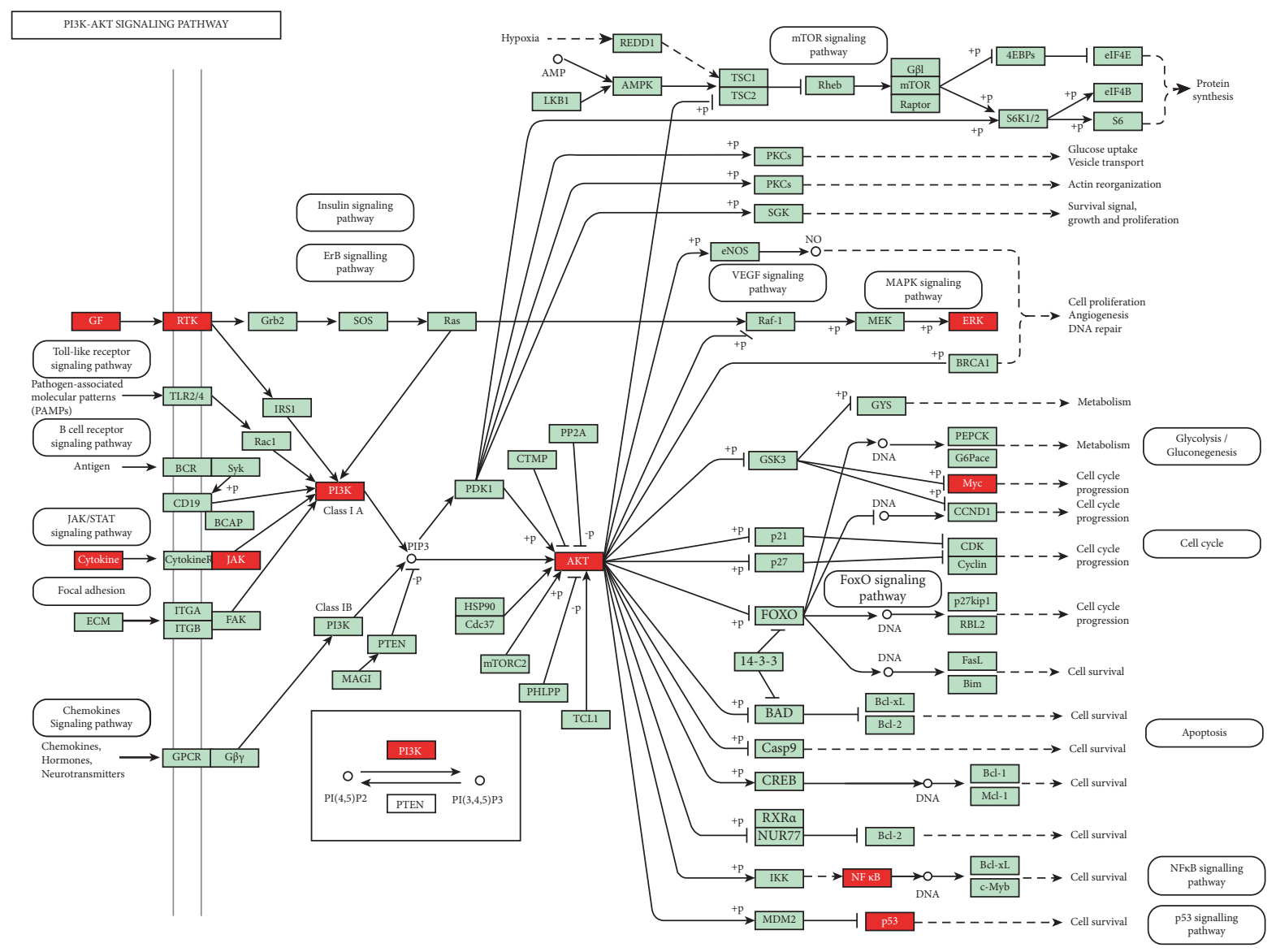

FIGURE 8: PI3K-Akt signaling pathway (KEGG). Red nodes represent the targets regulated by ZJP in UC. 


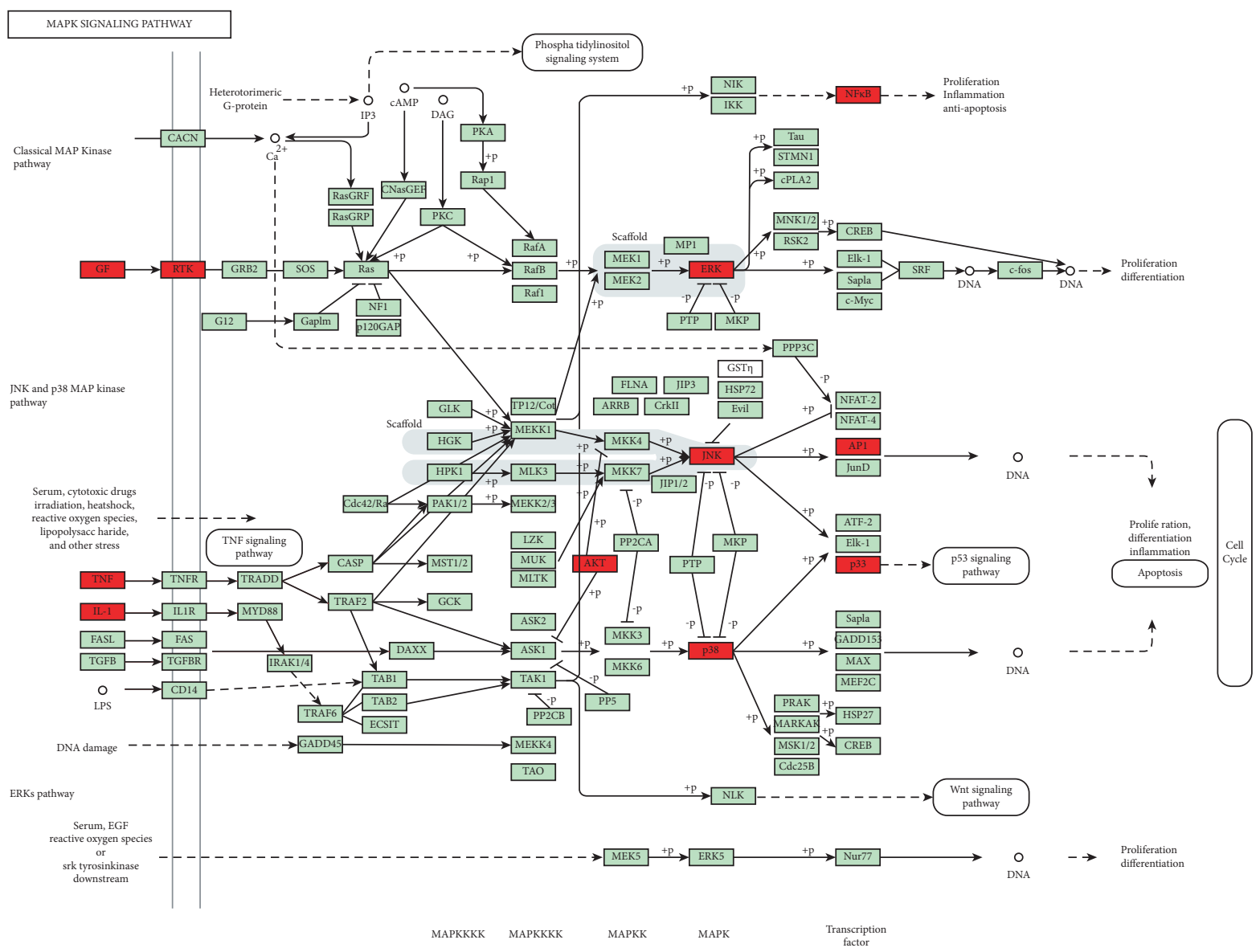

FIGURE 9: MAPK signaling pathway (KEGG). Red nodes represent the targets regulated by ZJP in UC.

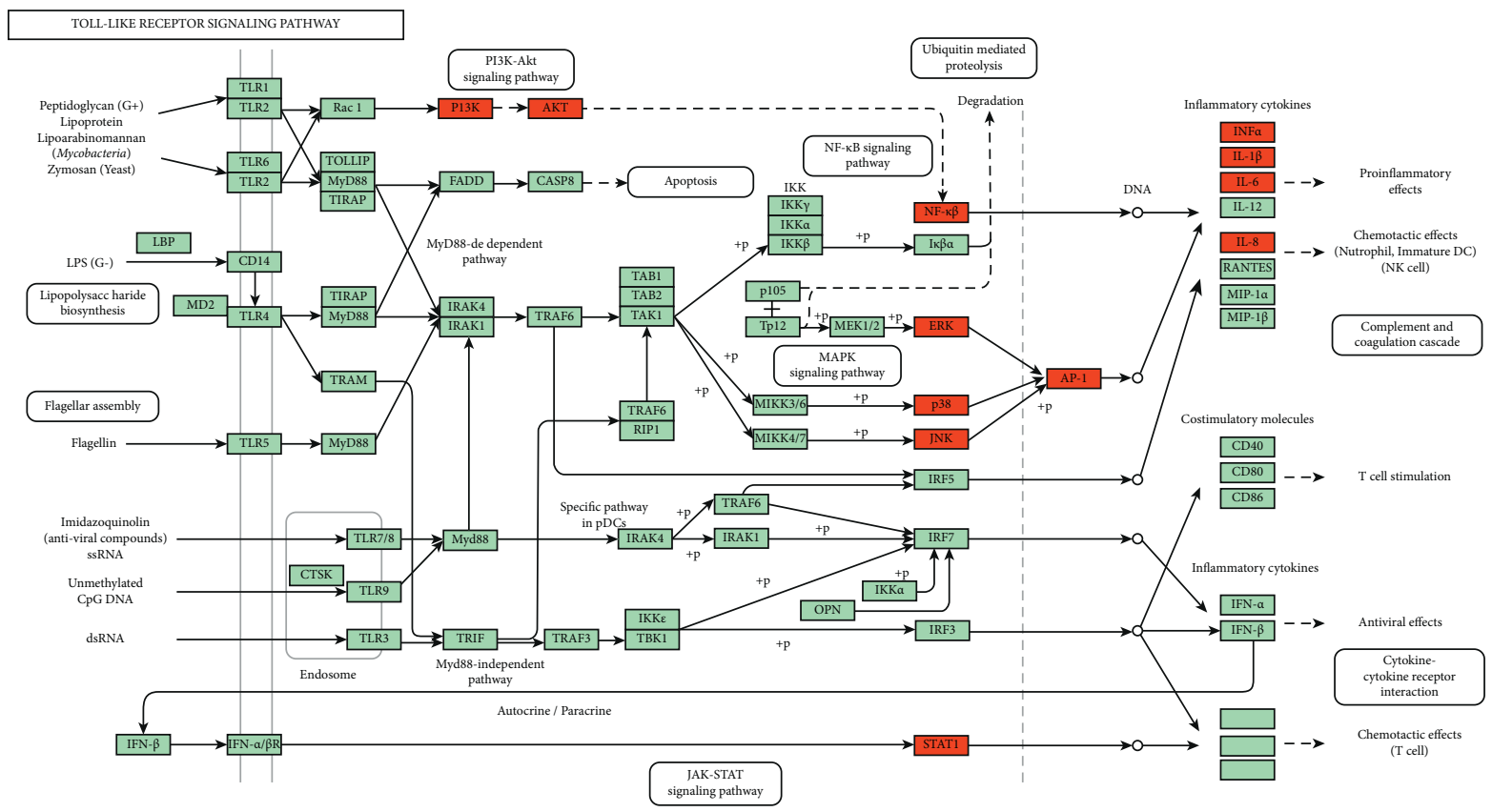

FIGURE 10: Toll-like receptor signaling pathway (KEGG). Red nodes represent the targets regulated by ZJP in UC. 
induced colitis via promoting mitophagy-mediated NLRP3 inflammasome inactivation [21]; $\beta$-Sitosterol could improve experimental colitis in mice by increasing the expression of antimicrobial peptides in intestinal epithelial cells [22]; isorhamnetin could ameliorate experimental IBD via PXRmediated upregulation of xenobiotic metabolism and downregulation of NF- $\kappa$ B signaling [23]; rutaecarpine could improve experimental colitis by regulating the synthesis and release of CGRP [24]. In general, most of these active ingredients could exert an anti-inflammatory response, regulate intestinal flora, and maintain the integrity of the mucosal epithelial barrier through the PI3K-Akt signaling pathway, MAPK signaling pathway, and toll-like receptor signaling pathway predicted in this study.

In addition to the abovementioned active ingredients with the research basis related to UC, five potential active ingredients including worenine, epiberberine, palmidin A, moupinamide, and rutalinidine have not been reported in UC. Recent studies have shown that worenine could reverse the Warburg effect and inhibit colon cancer cell growth by negatively regulating HIF- $1 \alpha$ [25]; epiberberine could be a novel antitumor candidate against $\mathrm{MKN}-45$-related gastric cancer via targeting the p53-dependent mitochondria-associated pathway [26]; moupinamide had anti-inflammatory potential found in Aswad's study [27]. Furthermore, there are few studies on palmidin $\mathrm{A}$ and rutalinidine, and the toxicity of rutalinidine was predicted to be low in this study, which was more worthy of further study. Precisely, because the effects and mechanisms of these potential active ingredients on UC have not been explained and verified, the research space and value are enormous. The abovementioned evidence preliminarily has suggested that these components of ZJP obtained by network pharmacology analysis may be effective components and jointly play anti-UC roles. These may be the material basis of ZJP in the treatment of UC, but further experimental verification and mechanism research are needed.

\section{Conclusions}

In short, researching ZJP on UC by use of the network pharmacological approach reflected the multicomponent, multitarget, and integrated regulation of TCM prescriptions. This study predicted the potential active ingredients, molecular targets, and mechanisms of ZJP in the treatment of UC, which provided a prospective scientific reference for the future systematic study of the effects and mechanisms of ZJP and its material basis on UC.

\section{Abbreviations}

ZJP: Zuojin pill

UC: $\quad$ Ulcerative colitis

TCMSP: Traditional Chinese Medicine Systems Pharmacology Database and Analysis Platform

CASC: Chinese Academy of Sciences Chemistry

OMIM: Online Mendelian Inheritance in Man

PPI: $\quad$ Protein-protein interactions

DAVID: The Database for Annotation, Visualization, and Integrated Discovery
KEGG: Kyoto Encyclopedia of Genes and Genomes

GO: $\quad$ Gene Ontology

ADME: Absorption, distribution, metabolism, and excretion

STRING: Search Tool for the Retrieval of Interacting Genes/Proteins

DL: Drug-likeness

OB: Oral bioavailability

BP: Biological process.

\section{Data Availability}

All data are available in the manuscript, and they are shown in figures and tables.

\section{Disclosure}

The paper has been preprinted (https://www.researchsquare. com/article/rs-317290/v1), but the authors have carefully modified it.

\section{Conflicts of Interest}

All authors of this manuscript do not have any conflicts of interest.

\section{Authors' Contributions}

Ying Wei conducted the analytical part, wrote the first version of the manuscript, and downloaded the reference and processed the graph and the table in the manuscript. Sichen Ren finalized the manuscript. Ruilin Wang, Manyi Jing, Honghong Liu, and Min Wang collected the data. Hongtao Song (corresponding author) and Yanling Zhao (corresponding author) conceived and coordinated the study and critically evaluated the data. Ying Wei and Sichen Ren contributed equally to this article.

\section{Acknowledgments}

The authors would like to thank the authors of all references. This study was financially supported by the National Key Research and Development Program (No. 2018YFC1704500).

\section{Supplementary Materials}

Table S1. Ingredients of each herb contained in ZJP. . (Supplementary Materials)

\section{References}

[1] I. Ordás, L. Eckmann, M. Talamini, D. C. Baumgart, and W. J. Sandborn, "Ulcerative colitis," The Lancet, vol. 380, no. 9853, pp. 1606-1619, 2012.

[2] S. C. Wei, J. Sollano, Y. T. Hui et al., "Epidemiology, burden of disease, and unmet needs in the treatment of ulcerative colitis in Asia," Expert Review of Gastroenterology \& Hepatology, vol. 1, pp. 1-15, 2021.

[3] P. Sehgal, J.-F. Colombel, A. Aboubakr, and N. Narula, "Systematic review: safety of mesalazine in ulcerative colitis," 
Alimentary Pharmacology \& Therapeutics, vol. 47, no. 12, pp. 1597-1609, 2018.

[4] M. Koning, R. Ailabouni, R. B. Gearry, C. M. A. Frampton, and M. L. Barclay, "Use and predictors of oral complementary and alternative medicine by patients with inflammatory bowel disease," Inflammatory Bowel Diseases, vol. 19, no. 4, pp. 767-778, 2013.

[5] A. V. Weizman, E. Ahn, R. Thanabalan et al., "Characterisation of complementary and alternative medicine use and its impact on medication adherence in inflammatory bowel disease," Alimentary Pharmacology \& Therapeutics, vol. 35, no. 3, pp. 342-349, 2012.

[6] B.-G. Zhou, F.-C. Liu, H.-M. Zhao, X.-Y. Zhang, H.-Y. Wang, and D.-Y. Liu, "Regulatory effect of Zuojin Pill on correlation with gut microbiota and Treg cells in DSS-induced colitis," Journal of Ethnopharmacology, vol. 262, Article ID 113211, 2020.

[7] S.-Y. Xiang, J. Zhao, Y. Lu et al., "Network pharmacologybased identification for therapeutic mechanism of Ling-GuiZhu-Gan decoction in the metabolic syndrome induced by antipsychotic drugs," Computers in Biology and Medicine, vol. 110, pp. 1-7, 2019.

[8] B. Zhu, W. Zhang, Y. Lu et al., "Network pharmacology-based identification of protective mechanism of Panax Notoginseng Saponins on aspirin induced gastrointestinal injury," Biomedicine \& Pharmacotherapy, vol. 105, pp. 159-166, 2018.

[9] A.-P. Li, S.-S. He, W.-N. Zhang et al., "Exploration the active compounds of Astragali Radix in treatment of adriamycin nephropathy by network pharmacology combined with transcriptomic approach," Journal of Ethnopharmacology, vol. 258, Article ID 112537, 2020.

[10] B. Ye, H. Shen, Y. Lu, and Y.-q. Wang, "Clinical observations on 100 cases of ulcerative colitis treated with the method of clearing away heat, expelling dampness, promoting blood circulation and healing ulcer," Journal of Traditional Chinese Medicine, vol. 30, no. 2, pp. 98-102, 2010.

[11] X. L. Chen, Y. Wen, Z. C. Wu et al., "Development of a traditional Chinese medicine syndrome-specific scale for ulcerative colitis: the large intestine dampness-heat syndrome questionnaire," Evidence-Based Complementary and Alternative Medicine, vol. 2018, Article ID 4039019, 10 pages, 2018.

[12] M.-y. Li, H.-j. Luo, X. Wu et al., "Anti-inflammatory effects of huangqin decoction on dextran sulfate sodium-induced ulcerative colitis in mice through regulation of the gut microbiota and suppression of the ras-PI3K-Akt-HIF- $1 \alpha$ and NF- $\kappa$ B pathways," Frontiers in Pharmacology, vol. 10, p. 1552, 2020.

[13] B. Liu, X. Piao, W. Niu et al., "Kuijieyuan decoction improved intestinal barrier injury of ulcerative colitis by affecting TLR4dependent $\mathrm{PI} 3 \mathrm{~K} / \mathrm{AKT} / \mathrm{NF}-\kappa \mathrm{B}$ oxidative and inflammatory signaling and gut microbiota," Frontiers in Pharmacology, vol. 11, p. 1036, 2020.

[14] M. Zobeiri, S. Momtaz, F. Parvizi, D. Tewari, M. H. Farzaei, and S. M. Nabavi, "Targeting mitogen-activated protein kinases by natural products: a novel therapeutic approach for inflammatory bowel diseases," Current Pharmaceutical Biotechnology, vol. 21, no. 13, pp. 1342-1353, 2020.

[15] N. Kordjazy, A. Haj-Mirzaian, A. Haj-Mirzaian et al., "Role of toll-like receptors in inflammatory bowel disease," Pharmacological Research, vol. 129, pp. 204-215, 2018.

[16] Y. Zhang, J. Wang, D. Hou, S. Yan, and S. Dang, "To assess the effective and safety of berberine hydrochloride in ulcerative colitis," Medicine, vol. 99, no. 49, Article ID e23482, 2020.
[17] X. Luo, B. Yue, Z. Yu et al., "Obacunone protects against ulcerative colitis in mice by modulating gut microbiota, attenuating TLR4/NF- $\kappa \mathrm{B}$ signaling cascades, and improving disrupted epithelial barriers," Frontiers in Microbiology, vol. 11, p. 497, 2020.

[18] S. Habtemariam and A. Belai, "Natural therapies of the inflammatory bowel disease: the case of rutin and its aglycone, quercetin," Mini Reviews in Medicinal Chemistry, vol. 18, no. 3, pp. 234-243, 2018.

[19] Z.-H. Zhang, H.-J. Zhang, A.-J. Deng et al., "Synthesis and structure-activity relationships of quaternary coptisine derivatives as potential anti-ulcerative colitis agents," Journal of Medicinal Chemistry, vol. 58, no. 18, pp. 7557-7571, 2015.

[20] X.-T. Yu, Y.-F. Xu, Y.-F. Huang et al., "Berberrubine attenuates mucosal lesions and inflammation in dextran sodium sulfate-induced colitis in mice," PloS One, vol. 13, no. 3, Article ID e0194069, 2018.

[21] C.-T. Mai, M.-M. Wu, C.-L. Wang, Z.-R. Su, Y.-Y. Cheng, and X.-J. Zhang, "Palmatine attenuated dextran sulfate sodium (DSS)-induced colitis via promoting mitophagy-mediated NLRP3 inflammasome inactivation," Molecular Immunology, vol. 105, pp. 76-85, 2019.

[22] K. Ding, Y. Y. Tan, Y. Ding et al., “ $\beta$-Sitosterol improves experimental colitis in mice with a target against pathogenic bacteria," Journal of Cellular Biochemistry, vol. 120, no. 4, pp. 5687-5694, 2019.

[23] W. Dou, J. Zhang, H. Li et al., "Plant flavonol isorhamnetin attenuates chemically induced inflammatory bowel disease via a PXR-dependent pathway," The Journal of Nutritional Biochemistry, vol. 25, no. 9, pp. 923-933, 2014.

[24] D. N. Luo, F. J. Li, and Y. Y. Zou, "Therapeutic effects of rutaecarpine on dextran sodium sulfate-induced experimental colitis in mice," Zhonghua Yixue Zazhi, vol. 98, no. 7, pp. 533-538, 2018, in Chinese.

[25] L. Ji, W. Shen, F. Zhang et al., "Worenine reverses the Warburg effect and inhibits colon cancer cell growth by negatively regulating HIF-1 $\alpha$," Cellular and Molecular Biology Letters, vol. 26, no. 1, p. 19, 2021.

[26] M. Yu, L. Ren, F. Liang et al., "Effect of epiberberine from Coptis chinensis Franch on inhibition of tumor growth in MKN-45 xenograft mice," Phytomedicine, vol. 76, Article ID 153216, 2020.

[27] M. Aswad, M. Rayan, S. Abu-Lafi et al., "Nature is the best source of anti-inflammatory drugs: indexing natural products for their anti-inflammatory bioactivity," Inflammation Research, vol. 67, no. 1, pp. 67-75, 2018. 